Résumés des conférences et travaux

\title{
Méthodologie de la prosopographie à l'époque contemporaine
}

Isabelle Parizet

\section{OpenEdition}

\section{Journals}

Édition électronique

URL : https://journals.openedition.org/ashp/431

DOI : 10.4000/ashp.431

ISSN : 1969-6310

Éditeur

Publications de l'École Pratique des Hautes Études

\section{Édition imprimée}

Date de publication : 1 octobre 2008

Pagination : 231-237

ISSN : 0766-0677

\section{Référence électronique}

Isabelle Parizet, "Méthodologie de la prosopographie à l'époque contemporaine », Annuaire de l'École pratique des hautes études (EPHE), Section des sciences historiques et philologiques [En ligne], 139 | 2008, mis en ligne le 06 janvier 2009, consulté le 12 juillet 2021. URL : http://journals.openedition.org/ashp/ 431 ; DOl : https://doi.org/10.4000/ashp.431 


\title{
MÉTHODOLOGIE DE LA PROSOPOGRAPHIE À L'ÉPOQUE CONTEMPORAINE
}

\author{
Maître de conférences : $\mathrm{M}^{\text {me }}$ Isabelle PARIZET
}

Programme de l'année 2006-2007 : I. Sculpture et architecture. - II. Sources d'inspiration. III. Notices prosopographiques sur les sculpteurs de façades et ornemanistes au XIX ${ }^{e} s$.

Le temps de la formation étant achevé, qu'il soit simple ouvrier ou sorti de l'École des beaux-arts, le sculpteur d'ornements entrait rapidement dans la vie active, confronté aux exigences du métier. Son travail était tout d'abord subordonné aux lois propres à la sculpture monumentale : localisation sur la façade, orientation cardinale, exposition à la lumière, angle de vue et dimensions déterminaient l'échelle, la pratique du relief (ronde-bosse ou bas-relief), la perspective ou les diverses corrections optiques. Ces variables se révélaient donc tout aussi importantes que la composition artistique ou la portée didactique ou allégorique de l'œuvre.

D'autre part, dans la mesure où l'ornement était destiné à la « sculpture de façade ", il n'existait plus en tant que tel mais intimement lié à l'architecture. En reprenant les diverses prescriptions et réglementations en matière de hauteur, de volume et de saillies, il apparut que la législation définissait non seulement la structure architecturale mais les surfaces à décorer, le relief toléré, et la dimension des saillies autorisées. Certains règlements imposèrent même le choix des motifs, leurs mesures et leurs cadences sur la façade.

Les termes employés dans les textes législateurs de l'époque allaient se montrer très révélateurs de la hiérarchie accordée aux deux arts. Les premiers parlent de « sculpture architecturale » : le décor sculpté y est donc considéré comme un élément supplémentaire, simple outil au service de l'architecture circonscrit dans les parties les moins résistantes, les surfaces « solides » étant réservées aux lignes de force. Jusqu'aux années 1875-1880, la réglementation architecturale légifère la décoration, et les ornements sculptés (distribués avec parcimonie sous la direction et le contrôle des architectes) ne sont là que pour souligner l'ossature de l'édifice et la mettre en valeur. On comprend donc mieux que le sculpteur ait été considéré comme un simple exécutant, la structure de la construction contenant en elle-même sa propre cohérence décorative : le fameux silence qui entoure pendant des décennies le travail des ornements de façade trouve vraisemblablement sa source dans ce rapport réglementé et codifié de l'architecture et de la sculpture.

Avec la fin du siècle, l'évolution des règlements de hauteur et la permission de saillies plus importantes consentent à s'émanciper du carcan imposé par des générations de législateurs et d'architectes : les sculpteurs redeviennent des partenaires à part entière. De « sculpture architecturale », les textes viennent à préférer les termes d'《 architecture sculptée » : l'édifice devient un support sur lequel est appliqué 
un décor. La sculpture évolue en art décoratif plaqué sur la façade, sans obligatoirement de lien interne avec la construction : on voit peu à peu apparaître des façades où des artistes exécutent des éléments sculptés (du simple motif de frise à la cariatide) sans souci de l'édifice sur lequel s'ancre leur œuvre. La façade n'est plus qu'un lieu d'exposition, faire-valoir motif sculpté. L'ornement devient une œuvre à part entière, et l'architecture est reléguée à une place secondaire de simple support, au profit de la sculpture. Naissent alors de nouveaux risques : si chaque sculpteur décide de créer « son » œuvre, insouciant du concept d'ensemble, qu'advient-t-il de l'équilibre et de la synchronie entre architecture et sculpture?

Avec les années 1880 et le règlement des saillies de 1884, la façade connaît la profusion des motifs et la redondance des ornements, visibles tout particulièrement dans les façades d'inspiration éclectique. S'y ajoutent toutes les nouvelles ressources décoratives mises à la disposition de la fin du $\mathrm{XIX}^{\mathrm{e}} \mathrm{s}$. : l'amélioration de la fabrication, la mise aux points de nouveaux matériaux et de copies en série très fiables élargissent la palette du décor. Marbre, bronze, grès, céramique, mosaïque et terre cuite envahissent certaines façades (cf. les pavillons d'expositions universelles).

La sculpture architecturale a alors tendance à perdre sa fonction architectonique pour devenir purement décorative : elle emprunte aux matériaux leurs caractéristiques (veinures du marbre, fil de la pierre ou grain du bois y sont copiés et amplifiés) et à l'art décoratif ses motifs qu'elle transpose sur la pierre (les lambris habillent les panneaux lisses des murs, la flore déborde des chapiteaux, les consoles ne sont plus qu'anthropomorphes ou zoomorphes) : ainsi, un même élément de feuillage peut être traduit sur la pierre, le verre et le fer (cf. la maison Lalique, au 40, cours Albert- $\mathrm{I}^{\mathrm{er}}, 8^{\mathrm{e}}$ arr.). En outre, le travail est désormais exécuté en atelier et non plus sur le tas : cet abandon du chantier accentue le côté mobile de ces sculptures qui, matériellement, sont ajoutées à la façade : une abondante correspondance entre sculpteurs et architectes fait état du transport de ces pièces achevées en atelier, de leur pose, et enfin des heures de travail pour le raccord de ces éléments une fois placés.

Devant cette évolution radicale de la conception du décor sculpté, les contemporains n'ont pas manqué de réagir : si certains se réjouissent de voir leur liberté d'expression enfin reconnue, d'autres s'en inquiètent. Un article de G. M. Jacques publié dans L'Art décoratif en mai 1899 dénonce cette tendance à " décorer la façade » (cf. « Le modernisme dans l'architecture », p. 45-47). Pour lui, l'architecte

promène non le crayon mais le ciseau sur les masses, leur donne la façon, en fait surgir le détail, lequel est en même temps un décor [...] MM. de Baudot, Plumet, Schoellkopf font des façades satisfaisant aux règlements et qui ne donnent pas l'impression de platitude. Pourquoi? Parce qu'ils modèlent des masses comme faisaient les gothiques au lieu de s'ingénier à décorer une surface comme nous le faisons depuis Philibert Delorme... Et quelles décorations! On n'y sort de la morne banalité des faux pilastres ioniques, corinthiens, composites, de l'ennui des frontons de baies proposant une rangée de triangles à notre admiration, que pour tomber dans le solécisme saugrenu des clefs de fenêtres transformées en mascarons grimaçants (qu'est-ce que ces têtes là peuvent bien faire à cette place ?)... Des consoles [...] sont entortillées de couronnes sur lesquelles on cherche d'instinct les inscriptions "À mon époux ", "À notre tante », à moins qu'il n'en surgisse une ménagerie des lions de l'Atlas. Cela s'appelle donner l'expression, l'expression de quoi? 
La profusion d'éléments sculptés répandue sans grand discernement finit donc par dénaturer la composition architectonique : une façade comme celles de Lavirotte perd toute lisibilité structurelle alors que les immeubles de Viollet-le-Duc ( $c f$. le nº 28 de la rue Liège ou le $\mathrm{n}^{\circ} 15$, rue de Douai) frappent par la clarté de leur composition : ici, l'architecte a pris le parti de n'utiliser que quelques motifs simples (presque stylisés) dont le nombre restreint et l'emplacement délibéré ne sont là que pour permettre une lecture immédiate et intelligible des lignes de force de l'édifice. Car Viollet-le-Duc (ardant défenseur de «l'architecture pure ») incrimine violemment cette démesure du décor qui, sous une apparente richesse, cache une faille plus grave :

Il n'est pas de symptôme plus évident de la stérilité d'idées de l'architecte que l'abondance irraisonnée de la sculpture. L'ornementation sculptée est le meilleur moyen de dissimuler les défauts d'harmonie ou de proportions, qu'un embarras d'architecte. En occupant ou en croyant occuper ainsi le regard du passant, on dissimule des pauvretés ou des défauts choquants dans la composition, voire des maladresses et des oublis dans la structure (cf. Dictionnaire raisonné de l'architecture..., notice « Sculpture », p. 226).

L'ornementation sculptée des façades ayant été délibérément conçue pour être au service de l'architecture, il est logique et cohérent qu'à cette époque ce soit les architectes qui aient dessiné et dirigé les programmes de sculpture : l'examen des plans montre que l'emplacement, la nature, le dessin et les cotes des ornements à sculpter sont définis dès la demande de permis de construire par l'architecte lui-même (cf. la série V.O ${ }^{11}$ aux archives de Paris). Et la comparaison avec l'état actuel de la construction souligne la fidélité de l'exécution à ces dessins. Pour la sculpture statuaire, les architectes précisent même les mouvements, les attitudes, l'effet attendu : ainsi pour la statue de Nicolas Poussin destinée à la terrasse du palais du Louvre sur la place Napoléon, l'architecte Lefuel envoya au statuaire François Rude des indications qui ne laissaient guère d'alternatives au statuaire :

La statue de Nicolas Poussin que vous avez à traiter sera placée sur un piédestal porté par une colonne engagée, dont l'entablement sera de profil. La figure sera debout : elle aura de hauteur, y compris la plinthe, 2.99 à 3.00. La plinthe à $0.15,-$ de haut, sa face est de 1.00, l'épaisseur de 1.15 - Les pieds de la figure sont à 12.50 d'élévation au-dessus du sol de la place. La plus grande hauteur de banc de pierre étant de 1.00, il serait essentiel que sur sa face, la figure ne développât pas une dimension supérieure. Je crois également important de recommander beaucoup de calme et de la sobriété de lignes dans la composition (cf. Arch. nat. : 23 juin 1854, 64AJ 201).

Les indications sont d'ordre matériel (emplacement, taille, mesures, matériau, délai, crédit) maiselles précisent la portéee morale, le caractère édifiant ou l'effet instructif de l'œuvre. De même en 1897 pour les travaux du pont Alexandre III, le sculpteur Levasseur est recommandé par l'architecte Boileau qui souligne qu'il « sait faire la sculpture statuaire décorative dans un sentiment délicat et distingué très appréciable » (cf. Arch. nat. : F ${ }^{12}$ 4057). Il n'est donc pas rare de voir un sculpteur retenu pour sa capacité à exécuter les indications d'ordre psychologique données par l'architecte. La difficulté était de trouver une juste place entre une exécution reconnue mais totalement subordonnée à l'architecture, et une autre affirmant une liberté d'expression qui risquait de nuire à l'unité de l'édifice : la marge de manœuvre était mince et bien des 
sculpteurs de l'époque y échouèrent. Le tollé qui accompagna l'exécution de La Danse par Carpeaux au nouvel Opéra dépend pour une part de l'ambiguïté des liens de cette fin de siècle entre architecture et sculpture.

Pour endiguer la tentation constante des sculpteurs de façades à faire leur ouvre, les architectes eurent recours à plusieurs artifices : le tout premier reposait dans l'élaboration d'un cahier des charges extrêmement précis des travaux de sculpture (à Paris en l'espace de dix ans furent élaborés un Cahier des charges particulières, Direction des travaux du Service d'architecture du département de la Seine, 15 février 1866, $\mathrm{V} .42 \mathrm{M}^{1}$, un Cahier des charges générales d'architecture [applicables à la sculpture], Direction des travaux du Service d'architecture du département de la Seine, 16 octobre 1872, arch. de Paris V.7M ${ }^{1}$, et un Projet de Cahier des charges spéciales de sculpture d'ornements, préfecture du département de la Seine, sd [1880], ibid. V.1 M² 29). Ces documents énuméraient les conditions d'exécution et les clauses inhérentes aux travaux mis en adjudication. L'entrepreneur de sculpture rédigeait alors une soumission qui déterminait avec précision ses propres engagements : nature des travaux qu'il se proposait d'exécuter, matériaux employés (la nature de la pierre et son origine étaient souvent indiquées), délais d'exécution et prix. Lors de la signature du marché, il s'engageait de façon explicite à « suivre les indications données par l'architecte » : ce dernier lui fournissait des dessins extrêmement précis avec repères topographiques de l'emplacement de chaque ornement, cotes millimétrées et mesures. Toutes les indications de dimension étaient inscrites sur les croquis, élévations et coupes afin d'en permettre l'exécution. D'autre part, le sculpteur s'engageait « à ne pas sous-traiter le travail et à refaire les parties jugées insatisfaisantes jusqu'à l'accord de l'architecte ». Enfin, tout au long des différentes étapes de l'exécution, son travail était suivi de façon extrêmement rigoureuse par le maître d'œuvre lui-même, son inspecteur ou une commission de contrôle (cf. les journaux d'attachement, les registres d'ordres passés aux entrepreneurs et les rapports de visites).

La deuxième contrainte exercée sur les sculpteurs était l'usage de répartir le programme sculpté entre plusieurs exécutants : il est frappant de constater que les diverses fontaines monumentales de la seconde moitié du $\mathrm{XIX}^{\mathrm{e}} \mathrm{s}$. parisien, ayant toutes reçu une statuaire variée et abondante, ne sont jamais l'œuvre d'un seul artiste mais que le programme iconographique sculpté a été réparti entre plusieurs ténors : l'architecte Visconti a sollicité James Pradier et Gabriel Seurre pour la fontaine Molière de la rue de Richelieu (1844) et Davioud, quelques années plus tard, a fait travailler Francisque Duret, Eugène Guillaume, Elias Robert, Charles Gumery et Alfred Jacquemart à celle de la place Saint-Michel (1858-1860). On peut le constater également sur des façades privées, comme celle du Crédit Lyonnais boulevard des Italiens où dans les années 1882, pour la partie haute, Antonin Carlès, Henri Lombard, Edouard Pépin et Désiré Maurice Ferrary se sont partagés les quatre grandes figures allégoriques des Heures $d u$ jour alors qu'un cinquième sculpteur Camille Lefèvre exécutait le fronton où La Banque distribue les crédits, flanquée du Commerce et de l'Industrie, au bord de la Seine et du Rhône. Quant à la sculpture d'ornements, elle suit également cette propension au partage entre plusieurs bénéficiaires : en 1839-1840, la frise de la Maison Dorée boulevard des Italiens fut répartie entre les ornemanistes Jean-Baptiste Lechesne, JeanBaptiste Jules Klagman, Georges Jules Garraud et Pierre Louis Rouillard. Et au nou- 
veau Louvre, une adjudication concernant l'exécution de 40 chapiteaux composites de colonnes isolées du $1^{\text {er }}$ étage des pavillons sur les places Napoléon et du Carrousel fut signée le 2 août 1854 avec huit entrepreneurs d'ornements (Daillion, C. Lechevalier, Martin, Martrou aîné, Rousseau, E. Grandjeand, E. Court, et Cottebrune, cf. Arch. nat. : 64AJ 188).

Maintenu fermement d'une part par la législation, de l'autre par ce contrôle constant de l'architecte sur son travail, le jeune professionnel avait donc une marge d'expression extrêmement limitée. Son art résidait donc essentiellement dans sa pratique.

Comme il avait été analysé dans le séminaire 2006-2007, les écoles les plus prestigieuses (École des beaux-arts ou École spéciale de dessin, dite « Petite école ») formaient leurs élèves au dessin et au modelage; quant à ceux qui étaient rentrés directement dans un atelier, ils avaient été immédiatement confrontés à la pratique. Les chantiers de restauration (parisiens ou provinciaux) des édifices diocésains ou des bâtiments civils embauchaient largement, et bon nombre de jeunes sculpteurs des années 1850-1860 trouvèrent leur premier contrat dans des marchés d'estampages (cf. le sculpteur Revillon chargé au Vieux Louvre de l'estampage des motifs couronnant les fenêtres du rez-de-chaussée, Arch. nat. : 27 avril 1857, 64AJ 80 ou Rouillard qui signe un marché pour celui des 2 lions des fenêtres $d u 2^{e}$ étage du pavillon central, cf. Ibidem). Dessins, modelages, estampages leur apprenaient les motifs anciens où ils puisaient par la suite savoir-faire et inspiration.

Outre cet apprentissage sur le tas, ils pouvaient également recourir à la consultation des dessins élaborés par les étudiants de l'École des beaux-arts, celle des recueils d'architecture ou d'ornements (illustrés de planches gravées des relevés, détails, coupes et élévations), des albums de croquis cotés et des périodiques d'architecture. Ces nombreuses publications permettaient aux « compositeurs » du décor de façade (architectes et sculpteurs) de se familiariser avec l'architecture contemporaine et de trouver des sources d'inspiration en vue de l'exécution du décor sculpté qui leur incombait. Plusieurs séances du séminaire furent consacrées à l'étude de ces sources : le choix des planches, la variété des sujets abordés, la qualité du dessin, les détails et les précisions concernant tant l'architecture que la sculpture y ont été abondamment commentés.

Ce sont les architectes qui, les premiers, dressèrent des catalogues des maisons récemment élevées à Paris : chaque construction y était illustrée de plusieurs gravures et d'un commentaire succinct. La variété des planches permet de travailler sérieusement sur une construction : ainsi par exemple, les cotes relevées par Théodore Vacquer dans ses Maisons les plus remarquables de Paris (1860-70) indiquent avec minutie les détails mesurés et dessinés sur place de la composition architecturale comme du décor sculpté. D'autres ouvrages, plus généraux présentent un choix de façades modernes: les relevés y sont commentés, les planches parfois cotées. L'ensemble de ces divers ouvrages avaient le mérite de permettre de suivre « l'actualité architecturale » et de donner des exemples contemporains concrets qui pouvaient inspirer ou guider le dessin d'un modèle comme son exécution en pierre.

Avec le développement des procédés photographiques, une nouvelle série de recueils vit le jour à la fin du XIX ${ }^{\mathrm{e}} \mathrm{s}$. : grâce aux prises de vue, l'auteur pouvait exposer rapidement l'illustration d'un thème précis. Les pionniers en matière de photographie 
architecturale s'attachèrent aux vues générales d'un Paris très quotidien : immeubles de rapport et nouvelles constructions y sont saisis dans leur îlot, leur rue, leur cour. Ces ouvrages ont le mérite de replacer ces maisons dans leur environnement urbain. D'autres éditeurs s'attachèrent à montrer les édifices parisiens selon des séries thématiques : Paul Planat consacra un volume aux Maisons de rapport et un autre aux Hôtels privés (1890-1895). D'autres recueils s'attachèrent à l'étude d'un motif d'architecture, insistant sur le lien très complexe entre architecture et sculpture (cf. Motifs d'architecture et de sculpture du même Planat, Détails d'architecture avec Cent vingts consoles ou Cent portes cochères d'A. Cary [1903-1904], ou encore Portes modernes avec des détails d'architecture, ferronnerie et sculpture d'E. Rumler [1900]).

Une autre série d'ouvrages présentait les ornements classés par motifs de sculpture : les auteurs y recensaient l'application de cet élément dans un décor sculpté. Les quelques 360 livraisons des Matériaux et documents d'architecture d'A. Raguenet (réunies en feuillets classés par sujet) donnent une variation sur un thème choisi : Balcon, Porte, Console, Fronton, Angle, Chapiteau, Frise, Cartouche, Cariatide, Pilastre, Palme, etc. (le dessin y est précis mais non coté; les exemples sont puisés en majorité à Paris). Le sculpteur A. Carrier-Belleuse, lui, s'attacha à L'Application de la figure humaine à la décoration et l'ornementation (1884) après un première publication en 1866 d'Études de figures appliquées à la décoration. Quant à Henri Despois de Folleville et Victor Ruprich-Robert, ils étudièrent « 1'ornement par la Nature » : ils y recensaient les motifs végétaux, floraux ou fruitiers qui pouvaient servir de base à une transposition sculptée dans la pierre.

Il n'était pas inintéressant de signaler les études spécialisées sur un matériau particulier (cf. par exemple La Brique et la terre cuite publié par Pierre Chabat en 1881) ou sur une utilisation spécifique à but industriel (cf. Recueil de dessins pour l'art et l'industrie gravés par E. Collinot et Albert de Beaumont en 1859).

Les encyclopédies illustrées n'avaient pas le caractère systématique de certains recueils mais présentaient avantageusement un Choix de constructions récentes. De la même façon, les périodiques d'architecture eurent tous une rubrique concernant les dernières constructions élevées à Paris (cf. La Revue générale de l'architecture et des travaux publics publiée de 1840 à 1890, La Semaine des constructeurs de 1876 à 1898, La Construction moderne de 1885 à 1945) : les articles y offraient une description des constructions nouvelles (agrémentée de planches), parfois une étude technique des nouveaux procédés mécaniques, les difficultés rencontrées et, non moins précieux, une liste des collaborateurs intervenus sur le chantier.

Enfin, il est bon de ne pas négliger l'importance pédagogique et instructive des signatures sur les façades qu'apposaient architectes, sculpteurs et parfois entrepreneurs : elles permettaient à celui qui souhaitait travailler ou copier un motif ou une composition d'en connaître immédiatement l'auteur. Au 19, rue d'Aumale ( $\left(9^{\mathrm{e}}\right)$, le sculpteur A. Joussot a signé et daté son groupe d'angelots en 1854 alors que l'architecte a omis d'y apposer son nom. Au 51-51 ${ }^{\text {bis }}$, rue de Miromesnil $\left(8^{\mathrm{e}}\right)$, l'architecte Bury a fait intervenir le sculpteur Granet pour les cariatides soutenant le balcon du $2^{\mathrm{e}}$ étage de l'immeuble de rapport qu'il construit en 1883 : curieusement, l'artiste signe de façon quasi invisible en dessous de la plate-forme, sur le revers du balcon. 
$\mathrm{M}^{\mathrm{me}}$ Béatrice Etiennety est venue présenter sa recherche sur les maîtres d'œuvre des anthologies du Paris moderne de Normand : elle a ainsi fait bénéficier la conférence des différentes observations qu'elle a pu faire tant sur le choix des édifices sélectionnés que sur son impact dans la création architecturale de l'époque. Elle a également relaté les difficultés rencontrées lors de la localisation de certaines planches, illustré l'évolution de certains constructions (modifications, restaurations ou démolitions), et démontré les liens qu'elle a pu établir entre des maisons non identifiées et les recueils de Normand.

Les recherches de M. Ramette ont illustré le lien entre ces ouvrages de grande diffusion à visée pédagogie et le bénéfice que pouvait en tirer un jeune architecte. Lors d'une commande d'immeubles de rapport au début de l'avenue Victor Hugo (n ${ }^{\text {os }} 4-6$ ), Pierre Joseph Olive (originaire de Biot dans le Vaucluse et venu à Paris dans les années 1865) s'est effectivement largement inspiré des façades des hôtels de la place de l'Étoile dessinées par Rouhault de Fleury : l'examen attentif des relevés de Théodore Vacquer (cf. op.cit., planches 4 à 9) et leur comparaison avec les façades actuelles ont permis de retrouver la source de son inspiration. Une visite sur place a complété cette étude et facilité l'analyse des analogies entre les deux constructions. Elle a été suivie d'une autre investigation à une deuxième adresse exécutée par le même homme : l'immeuble de rapport à l'angle de la rue de Berne ( $\left.\mathrm{n}^{\circ} 2\right)$ et la rue Saint-Pétersbourg $\left(n^{\text {os }} 3-5\right)$ a montré comment l'architecte, confirmé par ce premier exemple, avait su en tirer parti et l'adapter à un autre programme plus restreint.

Enfin, une autre intervention a ouvert plus largement le champ de recherche sur les sources d'inspiration. À plusieurs reprises, notre recherche prosopographique avait recensé des italiens praticiens, metteurs aux points, ornemanistes, sculpteurs d'ornements ou sculpteurs-mouleurs présents sur les chantiers. Des échanges fructueux avec $\mathrm{M}^{\text {lle }}$ Barbara Musetti (doctorante en histoire de l'art sur « La réception de l'œuvre d'Auguste Rodin par les sculpteurs italiens du début du XX ${ }^{\mathrm{e}}$ siècle », dans une cotutelle entre les universités Paris IV-Sorbonne et Rome III) ont permis tout au long de l'année d'établir des liens entre des artistes venant de la région de Carrare et la sculpture parisienne. $\mathrm{M}^{\mathrm{lle}}$ Musetti est venue exposer quelques unes des pistes de sa recherche.

Au cours des séminaires, le travail sur les notices du Dictionnaire des sculpteurs de façades a été poursuivi : une présentation des éléments épars recueillis en majorité dans des sources manuscrites, des annuaires et almanachs de l'époque, des documents iconographiques et enfin des articles de presse contemporains, a permis de se familiariser avec le type de documents et d'informations collectés pour chaque sculpteur. Une première rédaction de la notice était alors fournie et retravaillée au séminaire : la fidélité de ce premier texte à la grille pré-établie lors du début de la recherche en était vérifiée, les lacunes étaient soulignées et les imprécisions résiduelles inventoriées. In fine, la dernière étape du travail consistait à en dégager la richesse prosopographique. À ce jour, 344 notices ont été rédigées pour les lettres A et B. 